\title{
Entropic-Based Separation of Diastereomers: Size-Exclusion Chromatography with Online Viscometry and Refractometry Detection for Analysis of Blends of Mannose and Galactose Methyl-a-pyranosides at "Ideal” Size-Exclusion Conditions
}

\author{
André M. Striegel ${ }^{1}\left[\right.$. Steven P. Trainoff ${ }^{2}$
}

Received: 21 May 2020 / Revised: 23 September 2020 / Accepted: 19 October 2020 / Published online: 7 November 2020

(c) The Author(s) 2020

\begin{abstract}
The separation of carbohydrate diastereomers by an ideal size-exclusion mechanism, i.e., in the absence of enthalpic contributions to the separation, can be considered one of the grand challenges in chromatography: Can a difference in the location of a single axial hydroxy group on a pyranose ring (e.g., the axial $\mathrm{OH}$ being located on carbon 2 versus on carbon 4 of the ring) sufficiently affect the solution conformational entropy of a monosaccharide in a manner which allows for members of a diastereomeric pair to be separated from each other by size-exclusion chromatography (SEC)? Previous attempts at answering this question, for aqueous solutions, have been thwarted by the mutarotation of sugars in water. Here, the matter is addressed by employing the non-mutarotating methyl- $\alpha$-pyranosides of D-mannose and D-galactose. We show for the first time, using SEC columns, the entropically driven separation of members of this diastereomeric pair, at a resolution of 1.2-1.3 and with only a $0.4-1 \%$ change in solute distribution coefficient over a $25^{\circ} \mathrm{C}$ range, thereby demonstrating the ideality of the separation. It is also shown how the newest generation of online viscometer allows for improved sensitivity, thereby extending the range of this so-called molar-mass-sensitive detector into the monomeric regime. Detector multidimensionality is showcased via the synergism of online viscometry and refractometry, which combine to measure the intrinsic viscosity and viscometric radius of the sugars continually across the elution profiles of each diastereomer, methyl- $\alpha$-D-mannopyranoside and methyl- $\alpha$-D-galactopyranoside.
\end{abstract}

Keywords Size-exclusion chromatography $\cdot$ Viscometry $\cdot$ Diastereomers $\cdot$ Carbohydrates $\cdot$ Refractometry

\section{Introduction}

Among liquid-phase separation methods, size-exclusion chromatography (SEC) is virtually unique in its ability to separate analytes based on their solution conformational entropy, $\Delta S$. Because of the direct relationship between $\Delta S$ and the flexibility of analytes in solution, SEC provides

André M. Striegel

andre.striegel@nist.gov

Steven P. Trainoff

strainoff@wyatt.com

1 Chemical Sciences Division, National Institute of Standards and Technology (NIST), 100 Bureau Drive MS 8390, Gaithersburg, MD 20899-8390, USA

2 Wyatt Technology Corp, 6330 Hollister Avenue, Santa Barbara, CA 93117-3115, USA a direct route for comparing to each other the flexibilities of monodisperse analytes, at identical solution conditions, when separation proceeds by an ideal, or near-ideal sizeexclusion mechanism. A principal application of SEC to the above effect, first introduced over a decade-and-a-half ago [1], has been to study how various parameters—-such as anomeric configuration, glycosidic linkage, degree of polymerization, linearity versus cyclicity, and intramolecular hydrogen bonding-quantitatively affect the solution flexibility of mono-, di-, and oligosaccharides, both at aqueous, quasi-physiological conditions of temperature and $\mathrm{pH}$ and in select organic solvents [2-8].

The importance of the above type of quantitation is noted in the way molecular flexibility imparted by di- and oligosaccharide anomeric configuration, and glycosidic linkage is responsible for differences in binding and docking of enzymes and bacterial toxins $[9,10]$, in aptameric binding 
specificity [11], and in so-called site-directed presentation, i.e., in the initiation of infection by bacteria and parasites through interaction with glycosylation sites on the surface of the proteins of the host organism [12]. For monosaccharides, anomeric differences have been found responsible for $\alpha$-glucose selectively binding sugar transport proteins over $\beta$-glucose [13], while diastereomeric configurational differences between the glucose, galactose, mannose, and talose carbohydrate moiety in C-linked antifreeze glycoproteins have been found to be essential to modulating recrystallization-inhibition activity [14].

While generally considered a fairly low-resolution separation technique, SEC benefits from the commercial availability of oligomeric columns with a low-molar-mass exclusion limit, meaning that the separation power can be concentrated into a fairly narrow molar mass range (as compared to spreading the separation power over a large range, necessary for determining, e.g., the broad molar mass distributions of many natural and synthetic macromolecules). Also, while practitioners are generally reminded that SEC separates on the basis of analyte size in solution (i.e., based on the hydrodynamic or solvodynamic volume of the analytes), the more fundamental thermodynamic reality is that it separates based on differences in the solution conformational entropy $\Delta S$ of the analytes $[15,16]$.

Monosaccharide diastereomers can be viewed as providing one of the grand challenges in SEC separation, i.e., can SEC separate two analytes of the same molar mass, same chemical formula, and essentially the same structure save for the axial versus equatorial orientation of a single hydroxy group on the sugar ring, and can it do so with minimal enthalpic contribution to the separation? While separation of sugar diastereomers by SEC and other liquid chromatography techniques has been shown for various aldohexoses and aldopentoses in aqueous solution $[4,6$, 17], mutarotation (i.e., interconversion between $\alpha$ and $\beta$ anomeric forms) in aqueous solutions and, even, in many organic solvents, means that each peak in a chromatogram generally corresponds to a heterogeneity of conformers in tautomeric and anomeric equilibrium with each other at the given solvent and temperature conditions. For example, Fig. 4 in [4] purportedly shows the baseline-level SEC separation of galactose and talose. However, the peak for talose is actually the peak for at least four major conformers in equilibrium with each other at the experimental conditions, because at $30{ }^{\circ} \mathrm{C}$ an aqueous solution of this monosaccharide is composed of $28.7 \%{ }^{4} C_{1} \beta$-pyranose conformer, $42.1 \%{ }^{4} C_{1} \alpha$-pyranose, $17.9 \%{ }^{3} E \alpha$-furanose, and $11.1 \%{ }^{3} E \beta$-furanose $[6,18]$. Likewise, hidden under the galactose peak in that same figure is an equilibrium solution composed of $64 \%{ }^{4} C_{1} \beta$-pyranose conformer, $30 \%{ }^{4} C_{1} \alpha$-pyranose, $2.5 \%{ }^{3} E \alpha$-furanose, and $3.5 \%{ }^{3} E \beta$ furanose [19]. (For both talose and galactose, the percent of acyclics present in solution is $<0.1 \%$ ).

To avoid mutarotation, sugars need to undergo chemical glycosylation at the anomeric carbon. (In the presence of an acid catalyst, aldoses and ketoses react with alcohols to form glycosides [20].) Most commonly, methyl glycosides are produced when the objective is to study monosaccharides without the complications of mutarotation. Here, we examine the methyl glycosides of $\alpha$-Dgalactose and $\alpha$-D-mannose, the structures of which are shown in Scheme 1. (The equilibrium composition of galactose in water is given above; for mannose, non-glycosylated $30{ }^{\circ} \mathrm{C}$ aqueous solutions are composed of $32.8 \%$ ${ }^{4} C_{1} \beta$-pyranose, $66.2 \%{ }^{4} C_{1} \alpha$-pyranose, and a $<1 \%$ combination of $\alpha$-and $\beta$-furanose isomers $[6,18]$.) As can be observed from Scheme 1, these two monosaccharides constitute a diastereomeric pair, the only difference between them being that in $\alpha$-MeMan the axial hydroxy group is located on carbon $2\left(\mathrm{C}_{2}\right)$, whereas on $\alpha$-MeGal the axial $\mathrm{OH}$ is located at $\mathrm{C}_{4}$. (The difference in the location of one hydroxy group, denoted by a red asterisk in Scheme 1, obviously corresponds to a two-stereocenter difference between these sugars.)

Given the incredibly small structural difference between $\alpha$-MeMan and $\alpha$-MeGal, SEC separation by an entropic mechanism would appear highly unlikely. However, with the advances in oligomeric SEC column technology over the last two decades, the availability of low-exclusion-limit columns with very clean particle surfaces means that the entire separation power can be concentrated in a low-molarmass region spanning only a few thousand $\mathrm{g} \mathrm{mol}^{-1}$ and that enthalpic contributions to the separation should be minimal.
Scheme 1 Structures of the diastereomers methyl- $\alpha$-Dmannopyranoside and methyl$\alpha$-D-galactopyranoside. Red asterisks denote location of the axial hydroxy group on each sugar

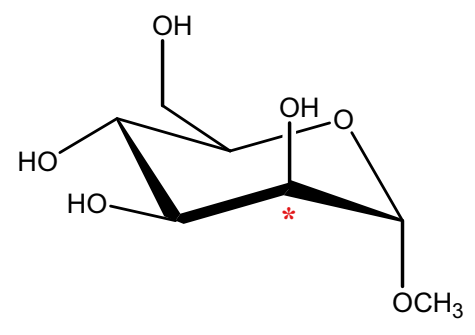

Methyl- $\alpha$-D-mannopyranoside ( $\alpha$-MeMan)

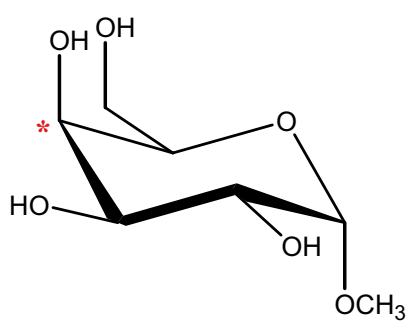

Methyl- $\alpha$-D-galactopyranoside ( $\alpha$-MeGal) 
The rationale behind our choice of detectors for this separation is discussed next.

\section{Combining Online Viscometry and Refractometry Detection}

The viscometer is generally considered a molar-mass-sensitive detector [21]. This is due to the fact that the viscometer responds to how an analyte enhances (generally, though a decrease, while rare, is also possible $[22,23])$ the viscosity of a solution vis-à-vis that of the neat solvent. Einstein's viscosity equation provides a direct link between the solution viscosity $\eta$ and the volume fraction $\phi$ of the solution occupied by the analyte [24]:

$\eta=\eta_{0}\left(1+2.5 \phi+4 \phi^{2}+\ldots\right)$,

where $\eta_{0}$ is the solvent viscosity. (It should be noted that the equation, as written, is for near-infinitely dilute solutions of hard spheres. Corrections for non-spherical geometries have been derived by Perrin, Guth, and Simha [25-27].) Given the generally accepted relation between $\phi$ and the hydrodynamic volume $V_{\mathrm{h}}$ of a molecule in solution, as per:

$\phi=\frac{c}{M} N_{\mathrm{A}} V_{\mathrm{h}}$,

where $c$ is the analyte concentration in solution and $N_{\mathrm{A}}$ is Avogadro's number, Eq. (1) can be written as:

$\eta=\eta_{0}\left(1+\frac{5}{2} \frac{c}{M} N_{\mathrm{A}} V_{\mathrm{h}}+\ldots\right)$.

From the above, it becomes clear why the sensitivity of a viscometer increases with an increase in hydrodynamic volume. The latter is related to molar mass via:

$V_{\mathrm{h}}=\frac{2[\eta] M}{5 N_{\mathrm{A}}}$,

with the intrinsic viscosity [ $\eta$ ] defined in Eq. (7) and accompanying discussion. This explains why the sensitivity of a viscometer increases with increasing molar mass $M$ [28]. At identical experimental conditions, large analytes will create a larger disturbance of flow as compared to their smaller counterparts. Consequently, the difference between the viscosity of a polymer solution and that of the neat solvent will be greater than will be the difference between an oligomer or monomer solution viscosity and the solvent viscosity. The viscometer, the operation and applications of which have been recently reviewed in this journal [29], is not a detector which is usually thought of as applicable in the study of oligomers or, much less, monomers. While exceptions to this can be found in the literature, these usually involve injecting fairly high oligomer loads onto the SEC column to obtain usable and reproducible chromatographic peaks [22, 23].
Recent work by our group has contradicted the above, demonstrating how an online differential viscometer can be used in the SEC study of various types of oligosaccharides spanning all the way from hexamers down to dimers [7]. It was noted that, in the oligomeric region, the viscometer can behave in a matter more akin to a concentration-sensitive detector than to a molar-mass-sensitive one. Shown here is that the newest generation of online viscometer is also capable of detecting monomers and that it does so with a sensitivity superior to that of earlier generations of the same type of instrument.

A differential refractometer was also part of the chromatographic setup, positioned following the differential viscometer in the detector train. A refractometer is a concentration-sensitive detector, its response DRI $\mathrm{I}_{\text {resp }}$ being proportional to the concentration $c$ of analyte in solution, as per $[15,30]$ :

$\mathrm{DRI}_{\text {resp }} \propto c \times \frac{\partial n}{\partial c}$,

with the parameter $\partial n / \partial c$ representing the specific refractive index increment of the solution at the solvent, temperature, and wavelength conditions of the experiment. The response of a viscometer, VISC $_{\text {resp }}$, is proportional $[15,29,31,32]$ to the specific viscosity $\eta_{\mathrm{sp}}$ of the solution, as per:

VISC $_{\text {resp }} \propto \eta_{\text {sp }}$.

In the case of both DRI and VISC, the proportionalities become equalities through the instrument constants for the particular pieces of hardware employed in the experiments.

From the above instrument responses, we see that the intrinsic viscosity $[\eta]$, defined as the ratio of the specific viscosity of an analyte solution to the concentration of analyte in the solution, in the limit of near-infinite dilution, as given by $[15,16,31]$ :

$[\eta] \equiv \lim _{c \rightarrow 0} \frac{\eta_{\mathrm{sp}}}{c} \propto \frac{\text { VISC }_{\text {resp }}}{\text { DRI }_{\text {resp }}}$

is obtained from the ratio of the responses of the viscometer and refractometer. For a chromatographic experiment employing both these detectors, as in the present case, $[\eta]$ can be obtained across the elution profile, for each chromatographic slice, subsequent to correction for interdetector delay and band broadening [33] if the detectors are connected in series (as done here), or to correction for eluent split ratio and column-to-individual-detector path length if the detectors are connected in parallel.

Determination of intrinsic viscosity provides an example of true detector synergism between the viscometer and the refractometer, as it is a parameter obtained by combining the information given by each detector, but which is not obtainable from either detector individually. Another example of 
viscometer-refractometer synergism is the determination of an analyte's viscometric radius $R_{\eta}$. This radius is defined conceptually as the radius of a homogeneous hard sphere (i.e., of a hard sphere of constant density and composition) which changes the viscosity of a fluid by the same amount as does the analyte, and defined mathematically as [15, 29, 31]:

$R_{\eta} \equiv\left(\frac{3[\eta] M}{10 \pi N_{\mathrm{A}}}\right)^{1 / 3}$,

where $M$ is the molar mass of the analyte and $N_{\mathrm{A}}$ is Avogadro's number.

The research presented herein aims to demonstrate that SEC separations performed under "ideal" conditions, i.e., in the virtually complete absence of enthalpic contributions to the separation, are both possible and can be employed for the near-baseline separation of monosaccharide diastereomers representing a single conformer in solution; and to further demonstrate that online viscometry is a sensitive means of detection in this monomeric regime. This work, employing multiple dimensions of detection $[15,16,31,50]$ rather than of separation [51, 52], serves to showcase the sensitivity of the latest generation online viscometer and its combined power with online refractometry in the monomeric region, as well as the virtually unique ability of SEC to separate diastereomers, at high resolution, through an "ideal," almost exclusively entropic mechanism.

\section{Experimental}

\section{Materials}

Methyl- $\alpha$-D-mannopyranoside (herein abbreviated as $\alpha$-MeMan) and methyl- $\alpha$-D-galactopyranoside (abbreviated as $\alpha$-MeGal) were both from Fluka Analytical (Milwaukee, WI) and sold as $>99.0 \%$ purity ( $\alpha$-MeMan) and as $>98.0 \%$ purity ( $\alpha-\mathrm{MeGal})$ by the manufacturer; their structures are shown in Scheme 1. Dextran $668 \mathrm{~K}$ (dextran with peak-average molar mass $M_{\mathrm{p}}$ of $\left.6.68 \times 10^{5} \mathrm{~g} \mathrm{~mol}^{-1}\right)$ was from Agilent/ Polymer Laboratories (Amherst, MA). Acetone and $\mathrm{NaN}_{3}$ were from Sigma-Aldrich (St. Louis, MO).

\section{SEC/VISC/DRI Analysis}

For the SEC/VISC/DRI experiments, $2 \mathrm{mg} \mathrm{mL}^{-1}$ solutions of each monosaccharide were prepared in deionized $\mathrm{H}_{2} \mathrm{O}+0.02 \% \mathrm{NaN}_{3}$, which also served as chromatographic mobile phase. Solutions were injected either individually or as mixtures, as described in "Results and discussion", at least in duplicate. The SEC system consisted of an Agilent 1260 isocratic HPLC pump (Agilent Technologies, Santa Clara, CA) with autosampler, a Waters 2695 column heater (Waters, Milford, MA), a column bank consisting of four $6 \mu \mathrm{m}$ particle size, $120 \AA$ nominal pore size, $7.8 \mathrm{~mm} \times 300 \mathrm{~mm}$ Ultrahydrogel Columns (Waters), a ViscoStar III differential viscometer (Wyatt Technology Corp., Santa Barbara, CA), and a T-rEX differential refractometer (Wyatt Technology Corp.). The vacuum wavelength of the light source in the refractometer was $658 \mathrm{~nm}$; the delay volume in the viscometer was set to $16.2 \mathrm{~mL}$. The ViscoStar III is a Wheatstone-bridge-type viscometer that benefits from patented auto-tuning for automatic balancing of the bridge [34] and patent-pending solvent compressibility correction to improve pump pulse rejection [35]. The SEC system was equipped with a 0.22$\mu \mathrm{m}$ nylon filter inline between the pump and the injector. The injection volume for each solution or mix was $400 \mu \mathrm{L}$. Flow rates and column and detector temperatures were as outlined in "Results and discussion". For experiments at $25{ }^{\circ} \mathrm{C}$, sample compartment, columns, and detectors were all maintained at this temperature. For experiments at $50{ }^{\circ} \mathrm{C}$, this denotes the column and detector temperatures, while the sample compartment temperature was $40{ }^{\circ} \mathrm{C}$ (this being the maximum temperature that the autosampler could achieve). Data acquisition and processing were performed with ASTRA (v. 7.3.2, Wyatt Technology Corp.).

The specific refractive index increment $(\partial n / \partial c)$ of each monosaccharide, a parameter which has been reviewed in this journal recently [36], was calculated based on the gravimetrically determined concentration of each individual monosaccharide solution and assuming $100 \%$ analyte recovery from the SEC columns. This assumption is supported by the purity and monodispersity of the sugars combined with the essential absence of enthalpic contributions to the separation and the equilibrium nature thereof (see "Results and discussion"). For $\alpha$-MeMan, the $\partial n / \partial c$ (in water at $25{ }^{\circ} \mathrm{C}$ and at a vacuum wavelength of $658 \mathrm{~nm}$ ) was determined to be $(0.1298 \pm 0.0008) \mathrm{mL} \mathrm{g}^{-1}$, while for $\alpha-\mathrm{MeGal}$ at the same experimental conditions the $\partial n / \partial c$ was $(0.1206 \pm 0.0001) \mathrm{mL} \mathrm{g}^{-1}$.

The chromatographic resolution $R_{\mathrm{s}}$ of blends was calculated using the Peak Fitting Module in OriginPro 2015 (OriginLab, Northampton, MA), using Eq. (9):

$R_{\mathrm{s}}=\frac{X_{\mathrm{c} 2}-X_{\mathrm{c} 1}}{0.5\left(w_{2}-w_{1}\right)}$,

where $X_{c 1}$ and $X_{c 2}$ are the peak centers of gravity and $w_{1}$ and $w_{2}$ are the constructed base widths, both based on a Gaussian fit of the chromatograms. 


\section{Results and Discussion}

\section{Separating Diastereomers by SEC}

Figure 1 shows the individual viscometer chromatograms of both monosaccharides, $\alpha$-MeMan and $\alpha$-MeGal, obtained at $25{ }^{\circ} \mathrm{C}$ and at a flow rate of $1 \mathrm{~mL} \mathrm{~min}{ }^{-1}$, overlaid upon each other. Given the small overlap between these peaks, separation of the diastereomers when these are in a mixture with one another appears likely. Indeed, as seen in Fig. 2, in a 1:1

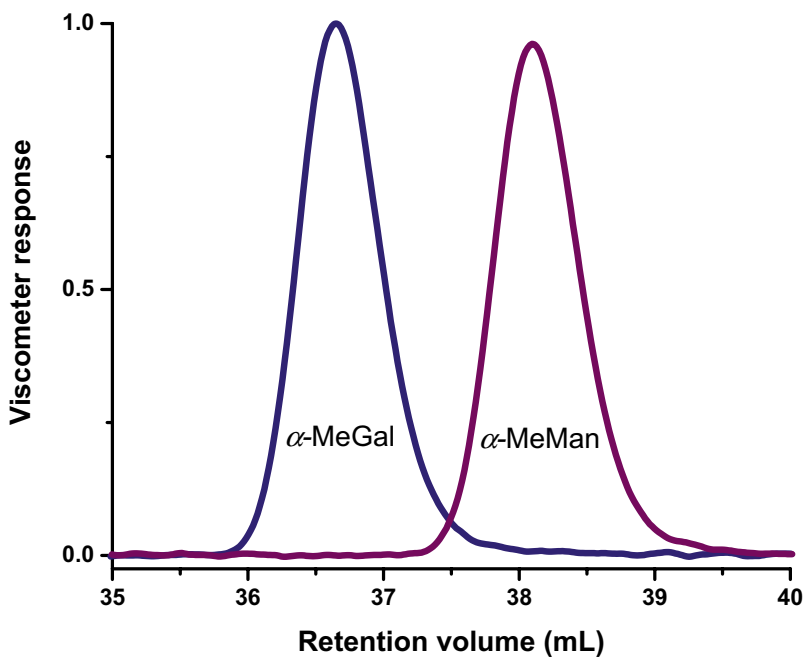

Fig. 1 Overlay of SEC/VISC traces of individual methyl- $\alpha$ glycosides, methyl- $\alpha$-galactoside ( $\alpha$-MeGal, blue trace), and methyl$\alpha$-mannoside ( $\alpha$-MeMan, purple trace), at $25{ }^{\circ} \mathrm{C}$ and $1 \mathrm{~mL} \mathrm{~min}^{-1}$ flow rate. See "Experimental" for details mixture at the same temperature and flow rate $\alpha$-MeMan and $\alpha$-MeGal separate with a chromatographic resolution $R_{\mathrm{s}}$ of 1.20. Overlaid upon this chromatogram in Fig. 2 are chromatograms of the same 1:1 mixture analyzed at the same temperature at flow rates of $0.5 \mathrm{~mL} \mathrm{~min}^{-1}$ and $0.25 \mathrm{~mL} \mathrm{~min}^{-1}$. As seen in Table 1 , as flow rate decreases, $R_{\mathrm{s}}$ increases. This indicates that we are operating in the $C$-term region of the van Deemter curve. Here, SEC separation is dominated by the $C_{\mathrm{SM}}$ subterm of the expanded van Deemter expression, corresponding to stagnant mobile phase mass transfer $[15$, 16]. We also note that increasing the temperature from 25 to $50{ }^{\circ} \mathrm{C}$ actually results in a decrease in $R_{\mathrm{s}}$, from 1.29 to 1.22 ; consequently, experiments were conducted at the lower temperature.

Given that only a very small gain in $R_{\mathrm{s}}$, from 1.29 to 1.32 , is achieved when decreasing the flow from 0.5 to $0.25 \mathrm{~mL} \mathrm{~min}^{-1}$ (meaning that we are approaching the van Deemter optimal flow rate $[15,37,38])$, but the experimental time is doubled in doing so, we opted for the former flow rate when performing experiments to evaluate the effect of temperature on chromatographic resolution, solute distribution coefficient, and to study mixture ratios. Running at $0.5 \mathrm{~mL} \mathrm{~min}{ }^{-1}$ provides a benefit additional to its rate-theoretical advantages. In general, the signal-to-noise ratio of bridge viscometers improves at higher flow rates. The reason for this is that, for a fixed capillary diameter, the strain rate applied to the sample increases in a manner directly proportional to the flow rate. With careful instrument design, the noise will be limited by either the stability of the pump or by the electronic noise in the measurement circuit. This study was performed using a new bridge viscometer design that
Fig. 2 Overlay of SEC/VISC traces of a 1:1 mix of $\alpha-\mathrm{MeGal}$ and $\alpha$-MeMan at (black) $1 \mathrm{~mL} \min ^{-1}, 25^{\circ} \mathrm{C}$; (red) $0.5 \mathrm{~mL} \mathrm{~min}^{-1}, 25^{\circ} \mathrm{C}$; (blue) $0.25 \mathrm{~mL} \mathrm{~min}^{-1}, 25^{\circ} \mathrm{C}$; (green) $0.5 \mathrm{~mL} \mathrm{~min}^{-1}, 50{ }^{\circ} \mathrm{C}$. See

"Experimental" for details

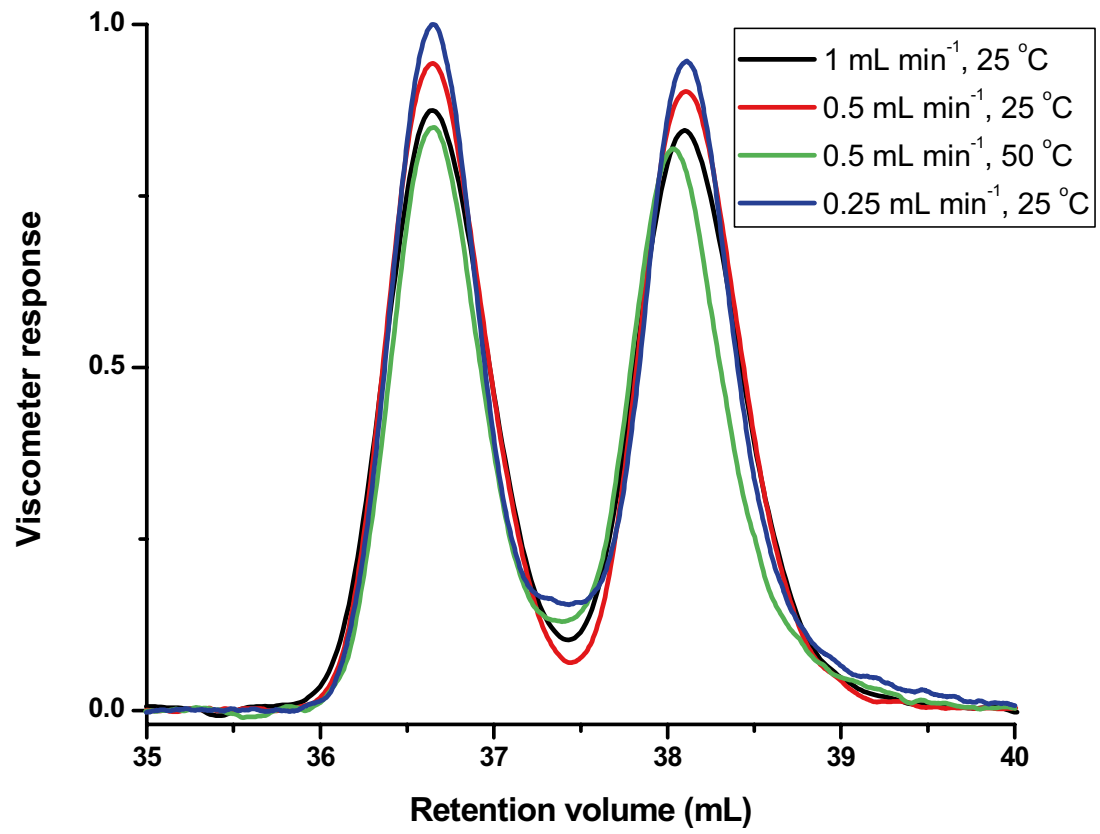


Table 1 Change in SEC resolution, $R_{\mathrm{s}}$, as a function of flow rate for separation of $\alpha$-MeGal and $\alpha$-MeMan

\begin{tabular}{|c|c|}
\hline Flow rate $\left(\mathrm{mL} \mathrm{min}^{-1}\right)$ & $R_{\mathrm{s}}$ \\
\hline 1.00 & 1.20 \\
\hline 0.50 & 1.29 \\
\hline $0.50\left(50^{\circ} \mathrm{C}\right)$ & 1.22 \\
\hline $0.50(3: 1 \mathrm{mix})$ & 1.10 \\
\hline 0.25 & 1.32 \\
\hline
\end{tabular}

All results for analyses of an $\alpha$-MeGal $/ \alpha$-MeMan 1:1 mix at $25{ }^{\circ} \mathrm{C}$, except where noted parenthetically. $R_{\mathrm{s}}$ was calculated employing Eq. (9) in text. In all cases, standard deviation is $R_{\mathrm{S}}< \pm 0.01$, with $n \geq 2$

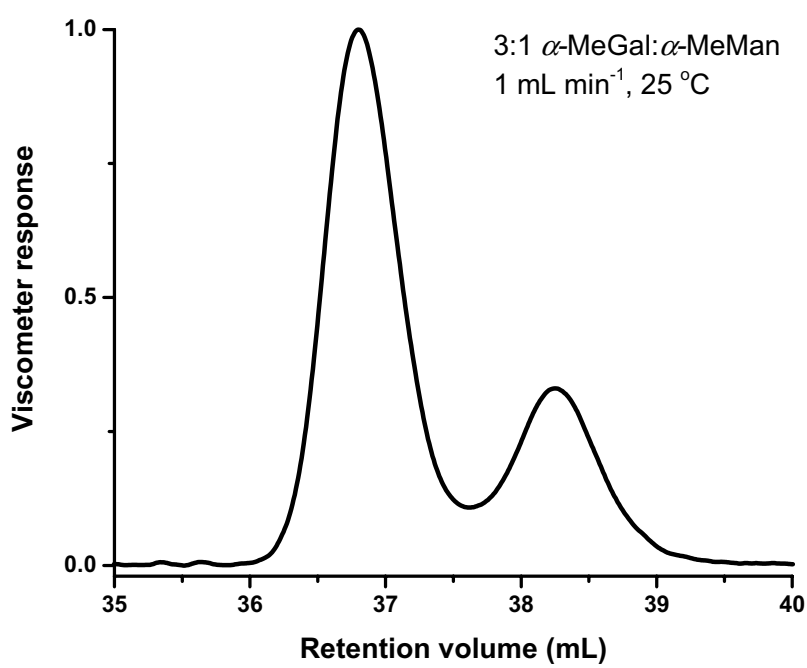

Fig. 3 SEC/VISC chromatogram of a 3:1 mix of $\alpha$-MeGal and $\alpha$-MeMan, obtained at $0.5 \mathrm{~mL} \min ^{-1}$ and $25^{\circ} \mathrm{C}$. See "Experimental" for details

corrects for compressibility effects in the instrument [35] so that resulting data were limited only by electronic noise. As a result, running at $0.5 \mathrm{~mL} \mathrm{~min}{ }^{-1}$ provides an additive benefit to the van Deemter advantages of doing so, to give the lowest noise data.

In comparison with the above results for $1: 1$ mixes, Fig. 3 shows the SEC/VISC chromatogram for a 3:1 mix of $\alpha$-MeGal $/ \alpha$-MeMan. As can be seen from Table 1 and when comparing this figure to the red trace in Fig. 2, the $R_{\mathrm{s}}$ of the 3:1 mix is lower than that of the $1: 1 \mathrm{mix}$ at the same flow rate and temperature, 1.10 versus 1.29. Again, while not baseline-resolved, the separation of the two diastereomers from each other is still quite remarkable given the large difference in their relative concentrations in the 3:1 mix.

\section{An Entropically Controlled Separation}

Having shown that the methyl glycoside diastereomers can be separated from each other, with good chromatographic resolution, using size-exclusion columns, it remains to be proven whether this separation actually occurs via a strict size-exclusion mechanism. The latter is defined as a separation in which the solute distribution coefficient, $K_{\mathrm{SEC}}$, depends only on the standard entropy difference between the phases, $\Delta S^{\circ}$, the two phases here being the flowing mobile phase outside the column pores and the stagnant mobile phase inside the pores $[15,16]$. In a so-called ideal size-exclusion separation, the standard enthalpy difference between the phases, $\Delta H^{\circ}$, does not contribute to the separation. As such, we can write [15]:

$K_{\mathrm{SEC}, \text { ideal }}=e^{\Delta S^{o} / R}$,

where $R$ is the gas constant. Equation (10) indicates that in an ideal SEC separation retention is temperature-independent.

Calculation of $K_{\mathrm{SEC}}$ is done using Eq. (11) [15]:

$K_{\mathrm{SEC}}=\frac{V_{\mathrm{R}}-V_{0}}{V_{i}-V_{0}}$,

where $V_{\mathrm{R}}$ is the retention volume of the peak maximum, $V_{0}$ the void volume of the columns (calculated using Dextran $668 \mathrm{~K}$; see "Materials"), and $V_{i}$ is the total column volume (calculated using acetone). From here, the solution conformational entropy $\Delta S$ of the analytes can be calculated via:

$\Delta S=R \ln K_{\mathrm{SEC}}$.

In reality, of course, there will always be some enthalpic contributions to the separation. Consequently, we speak of "near-ideal" SEC separations, generally understanding these to be SEC separations in which $K_{\mathrm{SEC}}$ shows little dependence on temperature, with changes in $K_{\mathrm{SEC}}$ on the order of less than $10 \%$ as a result of a $10{ }^{\circ} \mathrm{C}$ or more change in temperature [3-6].

Both methyl glycosides were analyzed at $25{ }^{\circ} \mathrm{C}$ and at $50{ }^{\circ} \mathrm{C}$, individually and also as a $1: 1 \mathrm{mix}$. Graphical results for the latter are shown in Fig. 2. Table 2 shows the results for the individual glycosides. (The negative sign on $\Delta S$ is due to the fact that solute permeation into the pores of the column packing material in SEC corresponds to a loss of conformational entropy [15].) As can be seen, over a $25^{\circ} \mathrm{C}$ change in temperature the change in $K_{\mathrm{SEC}}$ was only $1 \%$ for $\alpha$-MeMan and only $0.4 \%$ for $\alpha$-MeGal. We can conclude from these results that the SEC separation of these diastereomers proceeds by as close to an "ideal" size-exclusion, entropy-controlled mechanism as possible, i.e., with a virtually complete absence of enthalpic contributions to the separation. 
Table 2 Change in $K_{\mathrm{SEC}}$ and $\Delta S$ as a function of temperature for methyl glycosides

\begin{tabular}{|c|c|c|c|c|c|c|}
\hline & \multicolumn{2}{|l|}{$25^{\circ} \mathrm{C}$} & \multicolumn{2}{|l|}{$50^{\circ} \mathrm{C}$} & \multicolumn{2}{|l|}{$|\% \Delta|$} \\
\hline & $\Delta S\left(\mathrm{~J} \mathrm{~mol}^{-1} \mathrm{~K}^{-1}\right)$ & $K_{\mathrm{SEC}}$ & $\Delta S\left(\mathrm{~J} \mathrm{~mol}^{-1} \mathrm{~K}^{-1}\right)$ & $K_{\mathrm{SEC}}$ & $\Delta S$ & $K_{\mathrm{SEC}}$ \\
\hline Me- $\alpha$-mannopyranoside & -2.19 & 0.768 & -2.10 & 0.776 & 4.11 & 1.03 \\
\hline Me- $\alpha$-galactopyranoside & -2.85 & 0.709 & -2.88 & 0.706 & 1.05 & 0.423 \\
\hline
\end{tabular}

Flow rate, $0.5 \mathrm{~mL} \mathrm{~min}^{-1}$. All standard deviations $< \pm 1$ in the last significant digit, for $n \geq 3$. $K_{\mathrm{SEC}}$ and $\Delta S$ calculated employing Eqs. (11) and (12) in text, respectively
It should be noted that the almost complete independence of retention volume on either flow rate or temperature, as seen in Fig. 2, attests to the equilibrium nature of the SEC separation. (According to chromatographic theory, SEC retention is an equilibrium, entropy-controlled, temperature-independent process $[15,16])$. Chromatographic band broadening, of course, is neither temperature- nor flow-rate-independent, which is why differences in $R_{\mathrm{s}}$ are observed as a function of changes in these two parameters, even while the retention volumes of the individual analytes remain constant. The greater entropy of the galactoside over the mannoside, as seen in Table 2, appears due to the $\Delta 2$ effect [44], i.e., to the greater destabilization of the pyranose ring when the axial $\mathrm{OH}$ is located on carbon 2 [20, 44-48]. A more complete explanation of this effect and of its influence on the solution flexibility of monosaccharides can be found in references $[4,6]$.

\section{Detector Synergism: Online Viscometry and Refractometry}

For the present analytes, the intrinsic viscosity of each monosaccharide, defined as per Eq. (7), was measured at $25^{\circ} \mathrm{C}$ for individual runs at $1 \mathrm{~mL} \mathrm{~min}{ }^{-1}$, for $1: 1$ mixes at
$0.5 \mathrm{~mL} \mathrm{~min}^{-1}$ and $1 \mathrm{~mL} \mathrm{~min}-1$ and for the $3: 1 \alpha-\mathrm{MeGal} / \alpha$ MeMan mix at $0.5 \mathrm{~mL} \min ^{-1}$, with little variability among these. Figure 4 shows an overlay of the intrinsic viscosity of each monosaccharide upon an overlay of the viscometer and refractometer chromatograms, for a $1: 1 \mathrm{mix}$ at $25^{\circ} \mathrm{C}$ and $1 \mathrm{~mL} \min ^{-1}$. The $[\eta]$ of the two sugars are quite similar to each other, and, in both cases, there appears to be little variability in $[\eta]$ across the chromatograms, as expected for monodisperse species. It is also worth noting the little (essentially zero) distortion in peak shape as the chromatographic bands travel from the viscometer to the refractometer and within these detectors.

Calculation of the intrinsic viscosity is an example of true detector synergism. As seen from Eq. (7), $[\eta]$ is obtained from the ratios of the viscometer and refractometer signals after correction for interdetector delay and interdetector band broadening. This ratio can be done for each slice eluting from the columns, to map how $[\eta]$ changes (or not) across the chromatogram. Another example of how the information from viscometer and refractometer combines is given by the viscometric radius $R_{\eta}$, defined in Eq. (8) [49]. Here, the intrinsic viscosity just calculated is used in conjunction with the analyte molar mass, $194 \mathrm{~g} \mathrm{~mol}^{-1}$ for both $\alpha$-MeGal and $\alpha$-MeMan, to obtain $R_{\eta}$, one of several possible metrics
Fig. 4 Overlay of intrinsic viscosity $[\eta]$ (blue open circles) and viscometric radius $R_{\eta}$ (open magenta squares) upon an overlay of the viscometer (solid red line) and refractometer (solid green line) chromatograms of a 1:1 mix of $\alpha-\mathrm{MeGal}$ and $\alpha$-MeMan, at $25^{\circ} \mathrm{C}$ and $0.5 \mathrm{~mL} \mathrm{~min}^{-1}$. See "Experimental" for details

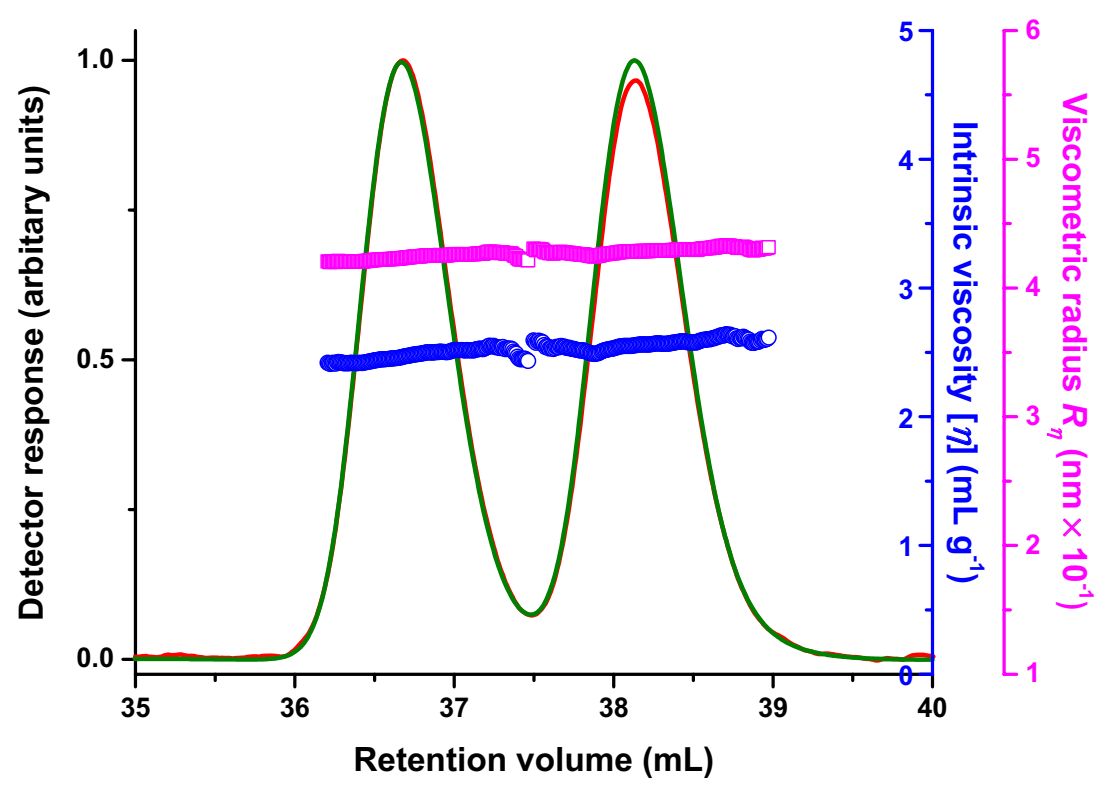


of analyte size in solution. As can be seen in Fig. 4, there is essentially no measurable difference in the $R_{\eta}$ of $\alpha$-MeMan and $\alpha$-MeGal, indicating that the present separation is not simply based on differences in solution size but rather, as explained earlier, on more fundamental solution conformational entropy differences.

The sensitivity of this new generation viscometer is seen to be superior to that of earlier generations: Previously, signal-to-noise $(S / N)$ ratios for even larger oligosaccharides (dimers, hexamers) were measured to be $\approx 15$ to 20 [7]. In the present case of $\alpha$-MeMan and $\alpha$-MeGal, for the same injection volume, flow rate, and number and type of columns, but twice the concentration as in our earlier oligosaccharide study ( $2 \mathrm{mg} \mathrm{mL}^{-1}$ here versus $1 \mathrm{mg} \mathrm{mL}^{-1}$ previous), we measured an $S / N$ of $\approx 120$ for both monosaccharides; i.e., the present generation viscometer employed herein appears to be 3 to 4 times more sensitive that previous viscometers of the same type, and certainly sensitive enough to allow for the study of not only oligomers and dimers but also, as demonstrated here, of monomers.

\section{Conclusions}

Demonstrated here is the size-exclusion chromatographic separation of a diastereomeric pair of non-mutarotating monosaccharides, methyl- $\alpha$-D-galactopyranoside, and methyl- $\alpha$-D-mannopyranoside, which differ from each other merely by the positioning of a single, axial hydroxy group. The separation was shown to proceed at equilibrium and by an "ideal" size-exclusion, entropy-controlled mechanism, essentially devoid of enthalpic interactions between the analytes and the column packing material. Chromatographic resolution of 1.2-1.3 was achievable with current oligomeric column technology coupled with the increased sensitivity associated with the latest generation differential viscometer. The latter, which is not normally considered adequate for the detection of small-sized, low-molar-mass analytes, is shown here to detect monomers with excellent signal-to-noise ratio.

This study serves to highlight the power of SEC in the oligomeric region, down to the monomeric regime. The ability to separate monodisperse analytes by an entropy-controlled mechanism allows quantitative study of their flexibility in solution, a parameter which, for mono- and higher-order saccharides, has been shown to influence enzymatic docking and binding, sweet response, the modulation of recrystallization-inhibition activity, etc. That the newest generation online viscometer can operate in this low-molar-mass range with superior performance allows not only for a new type of measurement for monomers but also for additional knowledge to be obtained about these analytes by combining the information gleaned from the viscometer with that from other types of detectors such as refractometers. Multidimensional detector synergism between viscometric and refractometric detection is showcased here via measurement of the intrinsic viscosities and viscometric radii of the two monosaccharides, across the chromatograms, when examined either individually or as part of a mix of the two sugars. Adding either a static or dynamic light scattering photometer to this detector train can provide additional advantages by way of direct measurement of molar mass and of information related to other size parameters, such as the hydrodynamic or Stokes radius, among others.

Acknowledgements This work was performed under CRADA CN-17-0041.

\section{Compliance with Ethical Standards}

Conflict of Interest One author (Trainoff) is Chief Scientist at and a full-time employee of Wyatt Technology Corporation, the manufacturer of the ViscoStar instrument that was used for this work.

Ethical Approval The research presented here did not involve human participants and/or animals. Commercial products are identified to specify adequately the experimental procedure. Such identification does not imply endorsement or recommendation by the National Institute of Standards and Technology, nor does it imply that the materials identified are necessarily the best available for the purpose.

Open Access This article is licensed under a Creative Commons Attribution 4.0 International License, which permits use, sharing, adaptation, distribution and reproduction in any medium or format, as long as you give appropriate credit to the original author(s) and the source, provide a link to the Creative Commons licence, and indicate if changes were made. The images or other third party material in this article are included in the article's Creative Commons licence, unless indicated otherwise in a credit line to the material. If material is not included in the article's Creative Commons licence and your intended use is not permitted by statutory regulation or exceeds the permitted use, you will need to obtain permission directly from the copyright holder. To view a copy of this licence, visit http://creativecommons.org/licenses/by/4.0/.

\section{References}

1. Striegel AM (2003) J Am Chem Soc 125:4146-4148 (see Erratum in Striegel AM (2004) J Am Chem Soc 126:4740)

2. Boone MA, Striegel AM (2006) Macromolecules 39:4128-4131

3. Boone MA, Nymeyer H, Striegel AM (2008) Carbohydr Res 343:132-138

4. Buley TD, Striegel AM (2010) Carbohydr Polym 79:241-249

5. Striegel AM, Boone MA (2011) Biopolymers 95:228-233

6. Morris MJ, Striegel AM (2014a) Biopolymers 101:703-711

7. Morris MJ, Striegel AM (2014b) Carbohydr Polym 106:230-237

8. Morris MJ, Striegel AM (2014c) Carbohydr Res 398:31-35

9. Lindberg AA, Brown JE, Strömberg N, Westling-Ryd M, Schulz JE, Karlsson K-A (1987) J Biol Chem 262:1779-1785

10. Rockey WM, Laederach A, Reilly PJ (2000) Proteins 40:299-309

11. Yang Q, Goldstein IJ, Mei H-Y, Engelke DR (1998) Proc Nat Acad Sci USA 95:5462-5467

12. Carver JP (1993) Pure Appl Chem 65:763-770 
13. Patching SG, Henderson PJF, Herbert RB, Middleton DA (2008) J Am Chem Soc 130:1236-1244

14. Czechura P, Tam RY, Dimitrijevic E, Murphy AV, Ben RN (2008) J Am Chem Soc 130:2928-2929

15. Striegel AM, Yau WW, Kirkland JJ, Bly DD (2009) Modern sizeexclusion liquid chromatography, 2nd edn. Wiley, Hoboken

16. Striegel AM (2017a). In: Fanali S, Haddad PR, Poole CF, Riekkola M-L (eds) Liquid chromatography: fundamentals and instrumentation, 2nd edn. Elsevier, Amsterdam, pp 245-273

17. Fu X, Cebo M, Ikegami T, Lämmerhofer M (2020) J Chromatogr A 1620:460981

18. Drew KN, Kajicek J, Bondo G, Bose B, Serianni AS (1998) Carbohydr Res 307:199-209

19. Franks F (1987) Pure Appl Chem 89:1189-1202

20. Stoddart JF (1971) Stereochemistry of carbohydrates. Wiley, New York

21. Mourey TH (2004) Int J Polym Anal Charact 9:97-135

22. Chance RR, Baniukiewicz SP, Mintz D (1995) verStrate G, Hadjichristidis N. Int J Polym Anal Charac 1:3-34

23. Striegel AM, Alward DB (2002) J Liq Chromatogr Rel Technol 25(13-15):2003-2022 (See erratum in Striegel AM, Alward DB (2003) J Liq Chromatogr Rel Technol 26(1):157-158, in which there is a typo: The value of $\left[\eta_{w}\right]$ for PE 282 in TCB at $135{ }^{\circ} \mathrm{C}$ should be $+0.0036{\left.\mathrm{dL} \mathrm{g}^{-1}\right)}^{-1}$

24. Hiemenz PC, Lodge TP (2007) Polymer chemistry, 2nd edn. CRC Press, Boca Raton

25. Perrin F (1936) J Phys Radium 7:1-11

26. Guth VE, Simha R (1936) Kolloid Z 74:266-275

27. Simha R (1940) J Phys Chem 44:25-34

28. Ekmanis JL (1989) 1989 Int GPC Symp Proc, Waters, Milford, pp 1-25

29. Striegel AM (2016) Chromatographia 79:945-960

30. Wyatt PJ (1993) Anal Chim Acta 272:1-40

31. Striegel AM (2005) Anal Chem 77(5):104A-113A

32. Trainoff S (2018) US Patent 7,331,218

33. Trainoff SP (2013) US Patent 7,386,427

34. Trainoff SP (2007) US Patent 7213439

35. Trainoff SP (2018) US Patent application 20180188146
36. Striegel AM (2017b) Chromatographia 80:989-996

37. Poole CF (2003) The essence of chromatography. Elsevier, Amsterdam

38. Snyder LR, Kirkland JJ, Dolan JW (2010) Introduction to modern liquid chromatography, 3rd edn. Wiley, Hoboken

39. Karger BL, Snyder LR, Horvath C (1973) An introduction to separation science. Wiley, New York

40. Giddings JC (1965) Dynamics of chromatography. Marcel Dekker, New York

41. Giddings JC (1991) Unified separation science. Wiley, New York

42. Caltabiano AM, Foley JP, Striegel AM (2018a) J Chromatogr A 1531:83-103

43. Caltabiano AM, Foley JP, Striegel AM (2018b) J Chromatogr A 1532:161-174

44. Reeves RE (1950) J Am Chem Soc 72:1499-1506

45. Shallenberger RS (1982) Advanced sugar chemistry. AVI, Westport

46. Juaristi E, Cuevas G (1995) The anomeric effect. CRC Press, Boca Raton

47. Izydorczyk M (2005). In: Cui SW (ed) Food carbohydrateschemistry, physical properties, and applications. Taylor and Francis, Boca Raton, pp 1-65

48. Orkid C, Bergeron DE, Rincon L, Hudgens JW, Gonzalez CA (2008) J Chem Phys 129:045102

49. Smith MJ, Haidar IA, Striegel AM (2007) Analyst 132:455-460

50. Striegel AM (2005) Multiple detection in size-exclusion chromatography. In: ACS symposium series 893, American Chemical Society, Washington, DC

51. Pasch H, Trathnigg B (2013) Multdimensional HPLC of polymers. Springer, Heidelberg

52. Pasch H (2013) Polym Chem 4:2628-2650

Publisher's Note Springer Nature remains neutral with regard to jurisdictional claims in published maps and institutional affiliations. 